\title{
Spinal cord potentials in traumatic paraplegia and quadriplegia
}

\author{
E M SEDGWICK, E EL-NEGAMY, AND H FRANKEL
}

From the Wessex Neurological Centre, Southampton, The Higher Institute of Physical Therapy, University of Cairo, Egypt, and The National Spinal Injuries Centre, Stoke Mandeville Hospital, Stoke Mandeville

SUMMARY Cortical, cervical and lumbar somatosensory evoked potentials were recorded following median and tibial nerve stimulation in patients with traumatic paraplegia and quadriplegia. The isolated cord was able to produce normal potentials even during spinal shock if the vertical extent of the lesion did not involve the generator mechanisms. The cervical potentials showed subtle changes in paraplegia at Th5 levels and below. In high cervical lesions the early cervical potentials may still be present but the later potentials were absent or, in partial lesions, delayed.

The first attempt to record spinal cord potentials in humans was by Pool, ${ }^{1}$ who noted epileptic-like waveforms from the distal cord in a paraplegic patient. Sawa ${ }^{2}$ inserted bipolar electrodes into the cords of psychotic patients and noted spikes and waves which were probably injury potentials. Magladery $e^{2} a^{3}$ were able to identify root and spinal potentials from intrathecal electrodes in the lumbar region following tibial nerve stimulation. Recently it has become possible to record potentials from the skin surface which originate in the spinal cord following peripheral nerve stimulation. ${ }^{4-7}$ Potentials from the lumbar region have been identified as originating in the roots and cord by Dimitrijevic et al, ${ }^{7}$ Delbeke et $a l,{ }^{8}$ and El-Negamy and Sedgwick ${ }^{6}$ while those recorded from over the cervical area originate from the brachial plexus, ${ }^{9}$ the dorsal horn of the cervical spinal cord, ${ }^{6}$ and from more rostral structures.

This investigation was carried out with two aims. Firstly, to determine whether the spinal cord could produce potentials when isolated from rostral neuroaxis and secondly, to see if spinal cord potentials could be of any clinical use in the diagnosis and assessment of the spinal injury patient.

Electrophysiological methods have been used previously to judge the extent of spinal cord

Address for reprint requests: Dr EM Sedgwick. Wessex Neurological Centre, Southampton General Hospital, Tremona Road, Southampton SO9 $4 \mathrm{XY}$

Accepted 20 March 1980 injury and its prognosis in experimental animals ${ }^{10-15}$ and in man, ${ }^{16-18}$ but all these workers studied the cortical somatosensory evoked potential. The animal studies showed that, after spinal cord injury, the cortical somatosensory evoked potential may be preserved for a few minutes if the injury was mild, but then disappeared. Early recovery of the potential preceded good functional recovery but Singer et al ${ }^{10}$ claim good functional recovery in monkeys even when the cortical potential had been absent for as long as 19 days.

Experience with humans shows that cerebral evoked potentials may be absent or attenuated early after injury but a gradual return and normalisation of potentials from stimuli originating below the site of injury heralds good functional recovery before any significant clinical improvement occurs. ${ }^{16-18}$

There have been no previous studies of the spinal cord potentials after spinal injury in man although they have been used in the diagnosis of multiple sclerosis ${ }^{19}$ and they are of abnormal form in cervical spondylosis. ${ }^{20}$

\section{Methods}

The methods have been described in detail by El-Negamy \& Sedgwick. ${ }^{6}$ Briefly, the median nerve at the wrist or the tibial nerve at the popliteal fossa was stimulated at three times sensory threshold or sufficient to produce a moderate muscle twitch in the absence of sensa- 


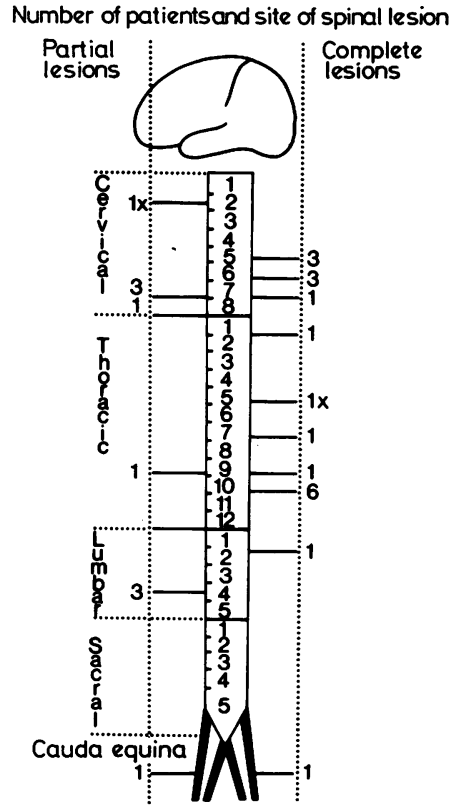

Fig 1 The number of patients and the level of the spinal cord lesion is shown diagrammatically. The patient marked $X$ had two lesions. Those with clinically complete lesions are shown on the right and those with partial or incomplete lesions on the left.

tion (about $2 \mathrm{x}$ motor threshold). Recordings from the skin overlying the vertebral column were amplified with a bandwidth of $10 \mathrm{~Hz}$ to $10 \mathrm{kHZ}$ and recorded on magnetic tape using a FM sys- tem with a bandwidth of 0 to $10 \mathrm{kHz}$. Off-line averaging by a PDP 12 computer produced the cervical or lumbar somato-sensory evoked potentials which were plotted and analysed.

Twenty-nine subjects aged between 19 and 66 years had all suffered traumatic complete or partial spinal cord section at the levels shown in fig 1. Two additional female patients aged 19 and 21 years had hysterical paraplegia and two others had cauda equina lesions, which were traumatic in one and a sequel to laminectomy performed at age three months for spina bifida in the other. Recordings were technically unsatisfactory from one other subject and he is excluded from this study. All but one were receiving inpatient treatment and all but one had recovered from spinal shock. Nineteen had received their injuries in the preceding eight months and the remainder two of 24 years earlier. They were taking their usual medication and were in good general health without pressure sores or urinary or other infection.

The recordist had no knowledge of the level or extent of the lesion and this information was not available until after the initial analysis of the results.

\section{Results}

Potentials from a normal subject are shown on the left side of fig 2. The upper trace shows the cortical potential recorded from $\mathrm{C} 3-\mathrm{Fz}(10-20$ system) after stimulation of the median nerve. The second trace, from electrodes placed over
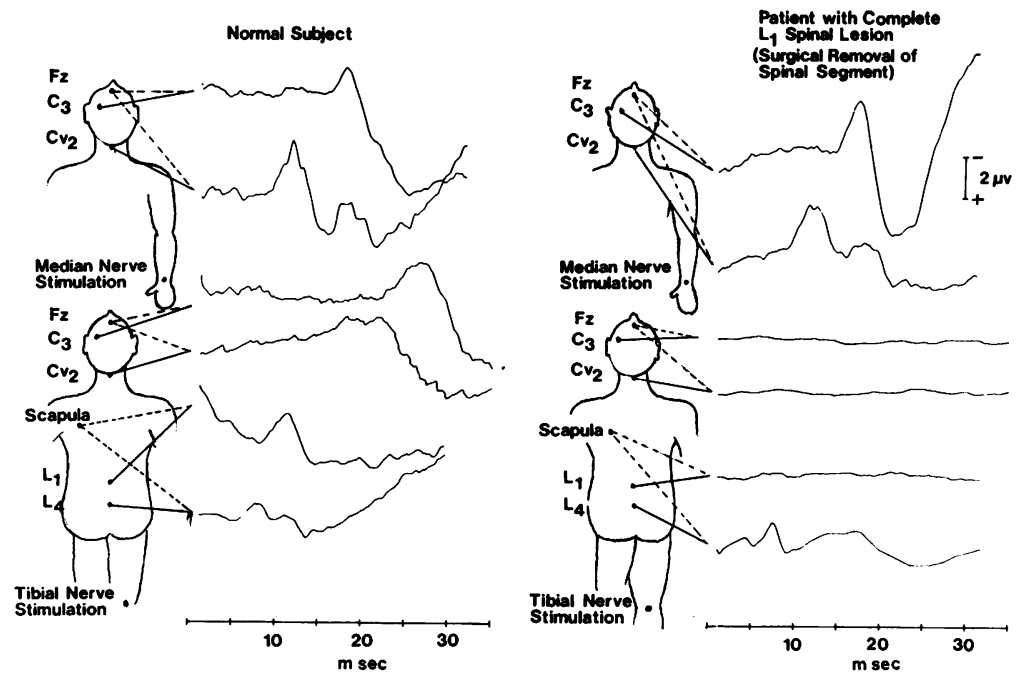

Fig 2 On the left are potentials from a normal subject following median nerve (upper 2 traces) and tibial nerve stimulation. The traces on the right are from a subject who had surgical removal of a damaged cord segment at L1. He had a normal N10 potential from L4 but no spinal or cortical potentials. Median nerve stimulation gave normal potentials. 
the 2 nd cervical vertebra $(\mathrm{Cv} 2)$ and at $\mathrm{Fz}$, shows the response to median nerve stimulation; four negative peaks, N11, N13, N14, and N20, named according to their polarity and approximate latency are evident. We follow the convention of denoting negativity at the "active" electrode by an upward reflection in the figures. The N20 potential is a cortical event and is best recorded by an electrode placed near the cortical sensory area as shown in the top trace. Tibial nerve stimulation at the popliteal fossa produced a double negative wave with peaks $\mathrm{N} 10$ and N14 from over the lumbar vertebrae, while only N14 is seen at $\mathrm{L} 1$ or lower thoracic levels. The Cv2Fz electrodes recorded a low amplitude, long duration potential of uncertain origin which has been studied by Jones and Small ${ }^{21}$ but not in detail by us. The scalp electrodes signal the arrival of the afferent volley at the cortex and the time delay from N10 is a measure of transmission time along the cord and through the sub-cortical structures.

The latencies and amplitudes of all these potentials were measured in all subjects and compared with recordings taken from 31 normal subjects in the case of the cervical potentials, and from 18 normal subjects in the case of the lumbar potentials. The amplitudes were much more variable than the latencies, but even the latencies showed considerable fluctuation due to different lengths of arms and legs. To overcome the problem of limb length, the latencies of the second and subsequent peaks were measured from the initial peak (N9) which indicates the arrival of the afferent volley in the brachial plexus. ${ }^{9}$ The N9 potential was recorded by electrodes on the 7 th cervical vertebra.

Spinal potentials caudal to the lesion

The tibial nerve contains afferents for L4 and $5, \mathrm{~S} 1$ and 2 and it is these spinal segments which give rise to the N14 lumbar potential. After spinal injury above this level the function of the caudal segments of the cord is severely impaired. Is there a similar impairment of the ability of the cord to produce the N14 potential?

Fig 2 shows the potentials recorded from a patient with a complete lesion of the cord at $\mathrm{L1}$. (The level given is the lowest segment with normal function.) The cervical and cortical responses to median nerve stimulation were normal but, as expected, no cortical or cervical potentials followed tibial nerve stimulation. A normal cauda equina or N10 potential was present at the L4 electrode, but no spinal or N14 potential at L1. This suggests that the isolated cord cannot produce potentials, but this patient had evidence of

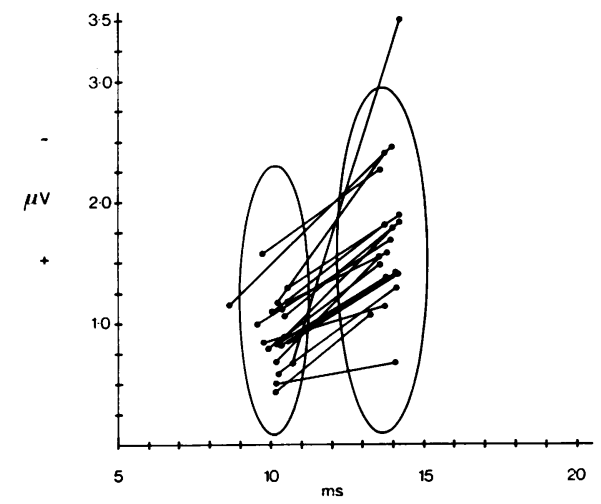

Fig 3 Amplitude and latency of N10 and N14 potentials following tibial nerve stimulation have been plotted from patients with lesions at Th10 and above. The ellipses represent the $90 \%$ limits for normal subjects. The one high amplitude N14 potential was recorded from a subject in spinal shock.

damage to the lower segments. The patient suffered root pains at the level of the transection and, in an effort to control them, the scar tissue in the spinal canal had been removed allowing an opportunity to confirm the completeness of his lesion. After operation, however, he lost a number of reflexes including his reflex bladder; it was concluded that further damage had been produced in the caudal segment of cord perhaps by interference with the blood supply. The absence of N14 in this patient was considered as due to this damage.

Other patients showed potentials of normal amplitude and latency from the caudal segment. In fig 3 the amplitude and latency of N10 and N14 for normal subjects have been plotted as ellipses so that the amplitude and latency points of $90 \%$ of normal subjects would be expected to fall within them. Fig 3 also shows the values from 19 patients who had two identifiable potentials and who had lesions at Th10 or above. It is of interest that the one point which lies beyond the normal range was obtained from a patient in spinal shock five days after a complete lesion at Th6.

Fig 4 shows the potentials from patients with lesions at Th10 and below. We have recorded a potential in a subject with a lesion as low as L3, but in other cases no potentials could be obtained.

We conclude that the isolated lumbar cord can produce normal potentials even though it lacks all descending control. Smaller isolated segments, 


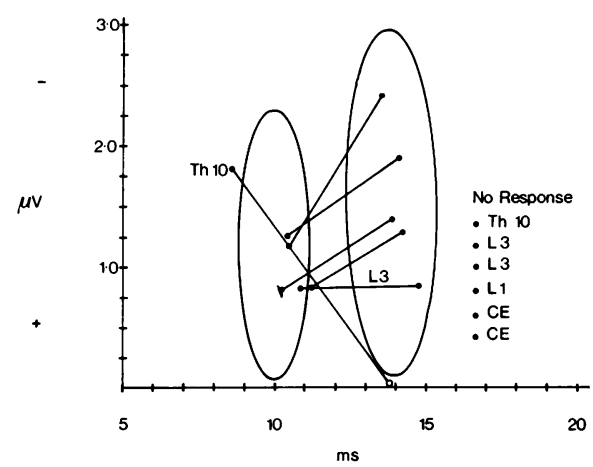

Fig 4 Amplitude and latency of the lumbar potentials in patients with lesions at Th10 and below. One patient had a lesion at L3 and gave normal potentials. Six other patients, listed on the graph, had no recognisable potentials. $C E=$ Cauda equina lesion.

due to lesions below Th10, were less able to produce recordable potentials. This could be because the generating segments were themselves involved in the lesion which usually extends over a few segments. The patients with cauda equina lesions showed no responses at all which was to be expected.

Spinal potentials rostral to the lesion In fig 5 the ellipses represent the $90 \%$ limits of the amplitude and latency of the cervical N11, $\mathrm{N} 13$ and N14 potentials of normal subjects. The latencies were computed from the peak of the N9 brachial plexus potential, which overcomes variability due to different arm lengths. The plotted points are from 17 patients with lesions at Th5 or below. The cervical potentials appeared normal (fig 2), but the points do not lie within the expected limits so a more detailed statiscal analysis was undertaken and the results

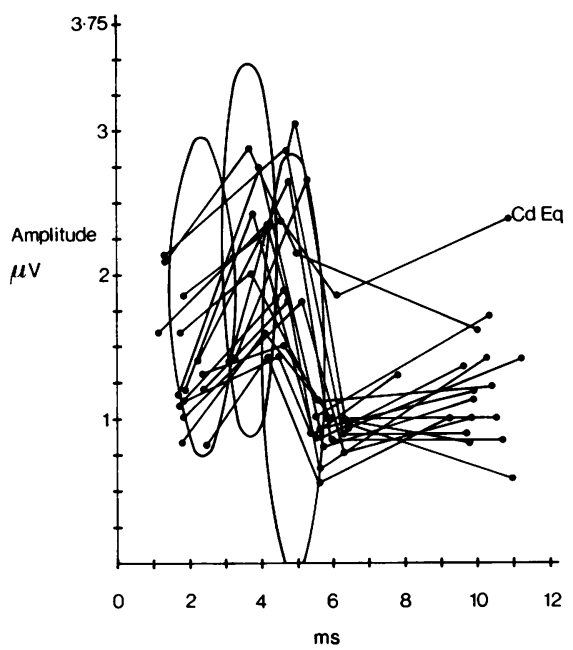

Fig 5 The ellipses represent the $90 \%$ limits of the amplitude and latency of the N11, N13 and N14 cervical somatosensory evoked potentials in normal subjects. Latencies were measured from the peak of the $N 9$ potential. The plotted points are from subjects with lesions at Th5 or below (including one with a cauda equina lesion). The final point, not encompassed by an ellipse, is of the N20 response.

are given in the table.

The table shows that the N9 potential was of the same amplitude in the paraplegic patients as in the normal subjects, but the latency was significantly shorter, $7.96 \mathrm{~ms}$ compared to $9.0 \mathrm{~ms}$ $(p=0.001)$. We believe this to be due to temperature differences in the laboratories where the studies were performed. The patients with spinal injury who cannot thermoregulate efficiently, were in a warm environment at about $24^{\circ} \mathrm{C}$ while the normals were studied in university laboratories which were not so well heated.

Table The cervical somatosensory potential from 31 normal subjects and 17 with spinal trauma below the Thl segment. The amplitudes of each component are given in $\mu V$ and the latentcy to peak from the preceding peak or from stimulus in the case of N9. Three normal subjects had no N9 component and one had no N14

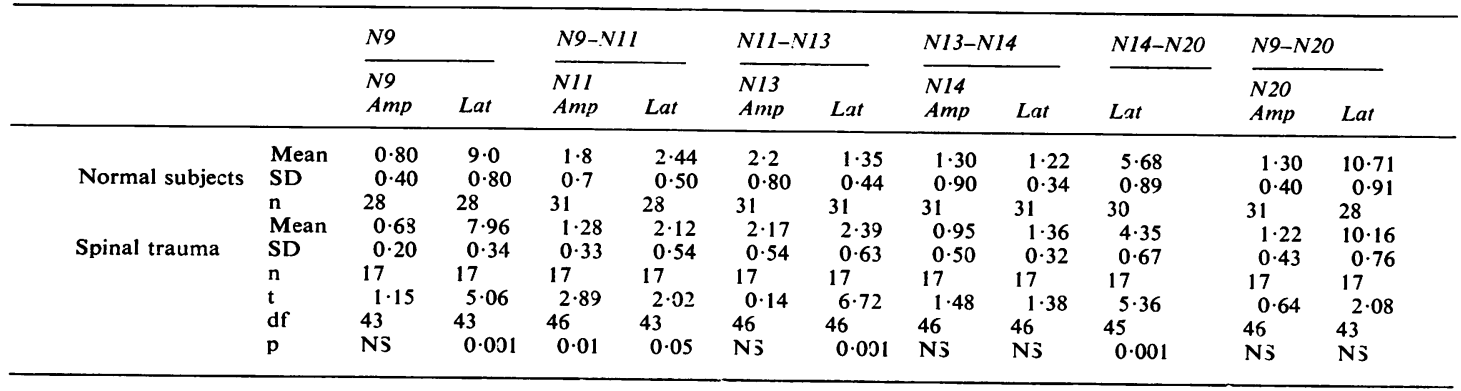


The amplitude of N11 was slightly less in the patients with spinal injury $(1 \cdot 28 \mu \mathrm{V}$ compared with $1 \cdot 8 \mu \mathrm{V})$, and the latency of the peak was also less $(2.12 \mathrm{~ms}$ compared with $2.44 \mathrm{~ms})$. The plotted points of N11 lie within or just to the left of the N11 ellipse.

The delay between $\mathrm{N} 11$ and N13 was prolonged by about $1 \mathrm{~ms}(2.39$ compared with $1.35 \mathrm{~ms}$ in the normal group). The amplitudes of the N13 potential were the same in both groups. The other difference between the groups was a shorter delay in the spinal injury group between the N14 and N20 potentials $(4.35 \mathrm{~ms}$ compared with $5.68 \mathrm{~ms}$ ). However, in spite of these changes the total conduction time from the $\mathrm{N} 9$ potential of the brachial plexus to the N20, the first cortical event, was the same in both groups.

The lengthening of the N11-N13 delay in spinal injury was an unexpected finding. N11 was thought to be generated in the dorsal horn of the cord, ${ }^{6}$ but our more recent work suggests that $\mathrm{N} 11$ may be presynaptic and that $\mathrm{N} 13$ is a postsynaptic dorsal horn neurone potential. Whatever the true origin of these potentials there seems to be some slight alteration in their generators after spinal injury some distance caudal to them. One explanation was that the degenerative and regenerative processes which follow trauma could produce a temporary change lasting perhaps seven months from the time of injury. This idea was tested by comparing the N11-N13 delay in 12 subjects who had suffered trauma in the previous seven months, and in whom the degeneration process may not have been totally completed, with five subjects whose trauma occurred at least 18 months before recording, allowing ample time for completion of the degeneration process. The N11-N13 delay was $2.40 \mathrm{~ms}$ for the recent trauma group and $2.16 \mathrm{~ms}$ for old trauma. Both groups were prolonged compared to normal and although the recently injured group showed a longer delay than the older group, the difference was not statistically significant.

\section{Patients with cervical lesions}

There were 13 patients with lesions at Th1 or above. Lesions in this region disturbed the cervical potentials as can be seen from fig 6 . Definite potentials could not be identified in seven patients. This may have been because the potentials were absent, but there were technical difficulties with recording from patients with high lesions; muscle relaxation was difficult to obtain (probably because they needed to use their accessory muscles of respiration even when lying

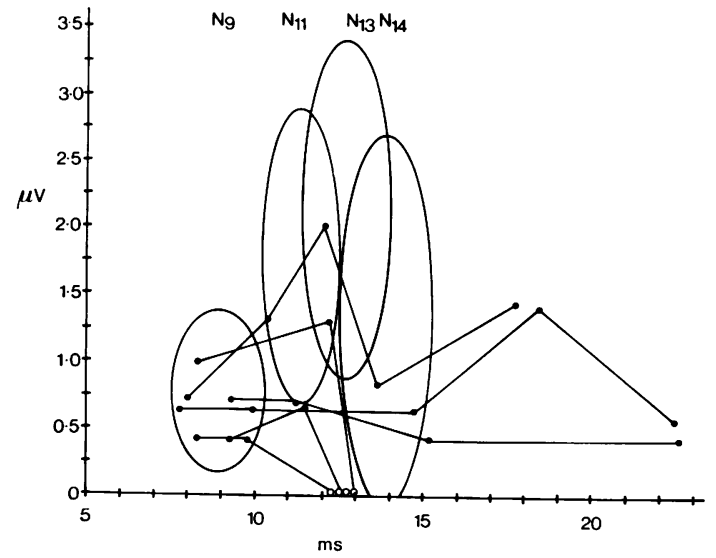

Fig 6 Plots of amplitude and latency of the cervical somatosensory evoked potential of patients with lesions at and above Th1. Latencies were measured from the point of stimulation (wrist) so that the N9 ellipse is included. This makes the $90 \%$ limits for N11, N13 and N14 broader to accommodate differences in arm length. Note the absence of later potentials in three cases and of the delayed potentials in two others.

still), and the EMG signal from these muscles may have swamped the potentials.

Of the five patients from whom potentials were recorded all had a normal N9. In three patients with complete lesions at Cv5 and Cv6 there was a recognisable potential of a latency approporiate for N11, but no subsequent potentials. One subject with a partial lesion at Th1, and another with a partial lesion at Cv7, gave small but recognisable N11 potentials but subsequent waveforms were delayed and the N20 appeared at $22.5 \mathrm{~ms}$, whereas it is normally seen at $19 \cdot 7 \mathrm{~ms}$.

\section{Spinal shock}

Only one subject with spinal shock was studied, but the observations seem worth recording. Fig 7 shows the lumbar potentials recorded five days after an injury which produced a complete lesion at Th6. At the time of recording he was completely areflexic and atonic with no bladder function or anal sphincter tone. Twelve days later the second recording was done at a time when reflex function was beginning to return. The N10 was of equal size on both occasions but the N14 was large when first tested but within normal limits on the second occasion.

\section{Discussion}

The N14 Lumbar potential is a segmental 


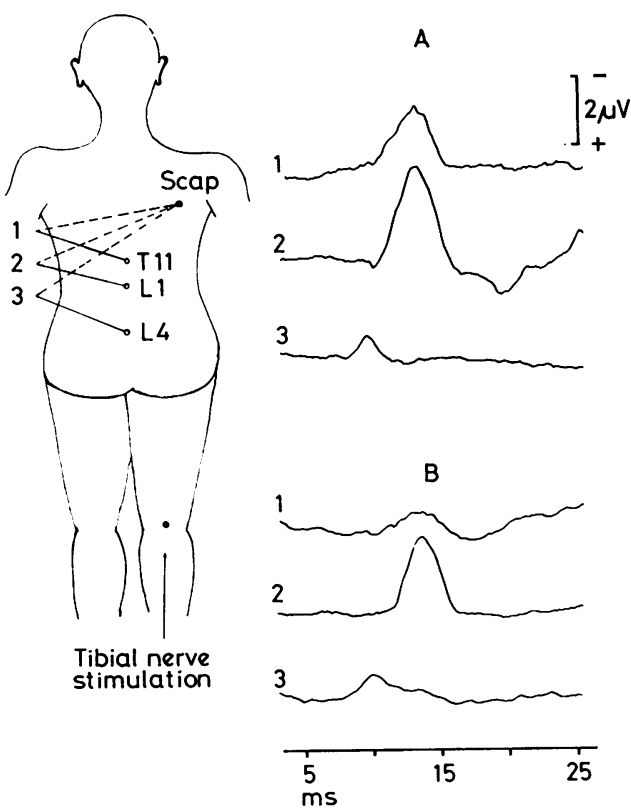

Fig 7 Lumbar somatosensory evoked potentials following tibial nerve stimulation in a patient in spinal shock. The potentials in A were obtained during the period of spinal shock, five days after the subject suffered a complete Th5 lesion. Those in $B$ were obtained 12 days later at a time when reflex function was beginning to return.

response $^{6}$ 7; Ertekin $^{22}$ has recorded a higher amplitude but similar potential using intrathecal electrodes. The available evidence suggests that the potential is postsynaptic and that it probably is generated in the dorsal horn of the spinal cord where low threshold afferent fibres synapse. The postsynaptic neurones in the dorsal horn are known to be under suppraspinal control and to alter their responsiveness in animals if the spinal cord is cooled or cut. ${ }^{23}$ So it was interesting to find a normal, Lumbar N14 potential in patients with spinal cord section (partial or complete) several segments rostral to the generator segments. This implies that the dorsal horn neurones are able to respond normally to an incoming volley. This is in contrast to the motor mechanisms whose function is severely disturbed after injury.

Lack of N14 from patients with lesions below Th10, and in some cases at Th10, could be due to the lesion extending several segments up and down the cord from the main point of impact. This is a common finding at autopsy but we have no anatomical knowledge of the extent of the lesions in our patients.
The presence of a potential corresponding to N11 recorded from Cv7 after median nerve stimulation has been interpreted as evidence that N11 is generated in the cord at segmental level," probably by dorsal horn synapses. Jones, ${ }^{9}$ however, suggested that $\mathrm{N} 11$ is a dorsal root or dorsal column potential and that N13 might be generated by segmental neurones. Further careful study of potentials in patients with high cervical lesions will help to resolve this point but good recordings from these subjects are technically difficult to obtain.

Finding minor abnormalities in the cervical potentials in patients with lesions at Th5 and below was unexpected. The rostral part of the cord is normally subject to some influences from caudal regions via the propriospinal system. The changes in potentials could be due to a reorganisation of synaptic inputs to neurones which lost some of their normal synapses due to degeneration. Such changes in experimental animals have been shown anatomically by Illis, ${ }^{24}{ }^{25}$ and functionally by Devor. ${ }^{26}$ An alternative explanation is that the degenerative process in the cord alters the micro-environment and produces slowed conduction in the remaining dorsal column fibres. Patients with old lesions (over 18 months), however, still showed an increased delay between N11 and N13. The N11N13 delay cannot be explained by the relatively early appearance of $\mathrm{N} 11$ in the spinal injury patients, for the N9-N13 time also is prolonged.

N14 is thought to be a potential generated in the thalamus and one would not expect a shortened delay between it and N20 which is the first cortical potential. The N9-N14 time is prolonged in the spinal injury patients but the shortened delay from N14-N20 allows some "catching up" so that N20 is at approximately the same latency in both groups when measured from N9.

Whatever the explanation of these changes, it is clear that disruption of the sensory pathway from the legs results in changes in the pathway from the arms. Perot ${ }^{16}$ noted attenuation of the median nerve cortical evoked potential for one week after spinal section, but he made no comment on latency changes (which appear quite marked in his figure 28.11) nor offered any explanation.

It is known from animal work that the caudal spinal cord normally influences the activity of rostral segments, an action often called the Schiff-Sherrington phenomenon. ${ }^{27}$ The altered cervical potentials may be a reflection of a similar process. This would be an interesting area for 
further investigation of the adaptation of the remaining CNS to a well-defined lesion.

\section{Clinical application}

Cortical potentials may be useful to determine the completeness of a lesion. The re-appearance of a cortical potential may herald clinical recovery, but their continued absence or marked abnormality is a bad prognostic sign. Cortical potentials were recorded in only three out of 11 cases in this series with clinically incomplete lesions, so the technique can demonstrate only the incompleteness of a lesion rather than prove the totality of transection. We would stress, however, that the experimental protocol was not the most appropriate to detect low amplitude, long duration and delayed potentials. Also we only stimulated the nerves on one side with a modest strength. Some simple modification to the technique may increase the yield. Nevertheless, at present the evoked potential technique is not as effective at demonstrating surviving ascending axons as are the techniques of Dimitrijevic et $a l^{28}$ for demonstrating descending ones. Further studies are needed for it is becoming clear that clinically complete lesions are not necessarily neurophysiologically complete. Demonstration of the survival of a few pathways may have therapeutic implications in the future.

Cortical and spinal potentials could have limited value in checking hysterical and malingering patients. Two hysterics, who had a paraplegia convincing enough to warrant their admission to a spinal injuries unit, both had entirely normal potentials. While such a finding does not exclude some degree of spinal injury, the discrepancies in neurophysiological and clinical findings could be helpful. These techniques could also be of use in assessing patients early after injury especially those with other injuries which preclude their co-operation in clinical examinations.

The potentials have no role in determining the level of a lesion. They could have a role however in assessing the longitudinal extent, especially of lower thoracic and high lumbar lesions where the integrity of the badder reflex mechanisms is in doubt. Also they could help in the assessment of high cervical lesions, when the survival of phrenic motoneurones is in doubt, and a decision on respiration by phrenic nerve stimulation has to be made. ${ }^{29}$

Finally, evoked potentials will play an important role in assessing spinal cord function during any therapy which is aimed at restoring spinal cord structure and function.
E El-Negamy was supported by the Arab Republic of Egypt.

\section{References}

1 Pool JL. Electrospinogram (ESG), Spinal cord action potentials recorded from a paraplegic patient. J Neurosurg 1945; 3:192-8.

2 Sawa H. Spontaneous electrical activities obtained from human spinal cord. Folia Psychiatr Neurol Jap 1947; 2:165-79.

3 Magladery JW, Porter WE, Park AM, Teasdale RD. Electrophysiological studies of nerve and reflex activity in normal man. IV: The two neurone reflex and identification of certain action potentials from spinal roots and cord. Bull John Hopkins Hosp 1951; 88:499-519.

4 Matthews WB, Beauchamp M, Small DG. Cervical somato-sensory evoked response in man. Nature 1974; 252:230-2.

5 Cracco RQ. Spinal evoked response: peripheral nerve stimulation in man. Electroencephalogr Clin Neurophysiol 1973; 35:379-86.

6 El-Negamy E, Sedgwick EM. Properties of a spinal somatosensory evoked potential recorded in man. J Neurol Neurosurg Psychiatry 1978; 41:762-8.

7 Dimitrijevic MR, Larsson LE, Lehmkuhl D, Sherwood A. Evoked spinal cord and nerve root potentials in humans using a non invasive recording technique. Electroencephalogr Clin Neurophysiol 1978; 45:331-40.

8 Delbeke J, McComas AJ, Kopec SJ. Analysis of evoked lumbosacral potentials in man. $J$ Neurol Neurosurg Psychiatry 1978; 41:293-302.

9 Jones SJ. Short latency potentials recorded from the neck and scalp following median nerve stimulation in man. Electroencephalogr Clin Neurophysiol 1977; 43:853-63.

10 Singer JM, Russell GV, Coe JE. Changes in evoked potentials after experimental cervical spinal cord injury in the monkey. Exp Neurol 1070; 29:449-61.

11 Croft TJ, Brodkey JS, Nulser FE. Reversible spinal cord trauma. A model for electrical monitoring of spinal cord function. $J$ Neurosurg 1972; 36:402-6.

12 D'Angelo CM, Van Gilder JC, Taub A. Evoked potentials in experimental spinal cord trauma. $J$ Neurosurg 1973; 38:332-6.

13 Deeke L, Tator CM. Neurophysiological assessment of afferent conduction in the injured spinal cord of monkeys. J Neurosurg 1973; 39:65-74.

14 Martin SH, Bloedel JR. Evaluation of experimental spinal cord injury. J Neurosurg 1973; 39: 75-81.

15 Cusik JF, Mkylebust JB, Larson SJ, Sances A. Spinal cord evaluation of cortical evoked responses. Arch Neurol 1979; 36:140-3.

16 Perot PL. Somatosensory evoked potentials in the evaluation of patients with spinal cord injury. 
In: Morley TP, ed. Current Controversies in Neurosurgery. Philadelphia: WB Saunders, 1976: 160-7.

17 Perot PL. The clinical use of somato-sensory evoked potentials in spinal cord injury. Clin Neurosurg 1973; 20:367-81.

18 Rowed DW, McLean JA, Tator CM. Somatosensory evoked potentials in acute spinal cord injury. Prognostic value. Surg Neurol 1978; 9: 203-10.

19 Small DG, Matthews WB, Small M. Subcortical somatosensory evoked potentials in multiple sclerosis. Electroencephalogr Clin Neurophysiol 1977; 43:536-7.

20 El-Negamy E, Sedgwick EM. Delayed cervical somatosensory potentials in cervical spondylosis. J Neurol Neurophysiol Psychiatry 1979; 42: 238-41.

21 Jones SJ, Small DG. Spinal and sub-cortical evoked potentials following stimulation of the posterior tibial nerve in man. Electroencephalogr Clin Neurophysiol 1978; 44:299-306.

22 Ertekin C. Comparison of the human evoked electrospinogram recorded from the intrathecal, epidural and cutaneous levels. Electroencephalogr
Clin Neurophysiol 1978; 44:683-90.

23 Wall PD. Dorsal Horn Electrophysiology. In: Iggo A, ed. Handbook of Sensory Physiology. Berlin: Springer, 1973: 253-70.

24 Illis LS. Experimental model of regeneration in the central nervous system I Synaptic changes. Brain 1973; 96:47-60.

25 Illis LS. Experimental model of regeneration in the central nervous system. II: The reaction of glia in the synaptic zone. Brain 1973; 96:61-8.

26 Devor M, Merrill EG, Wall PD. Dorsal horn cells that respond to stimulation of distant dorsal roots. J Physiol (Lond) 1977; 270:519-32.

27 Granit R. The basis of motor control. London: Academic Press, 1970: 180-3.

28 Dimitrijevic MR, Faganel J, Lehmkuhl D, Sherwood A. Motor control in man with spinal cord injury. In: Desmedt JE, ed. Motor control in man: suprasegmental and segmental mechanisms. Progress in Clinical Neurophysiology, vol 8 . Basel: Kargel; in press.

29 Glenn WWL, Holcomb WG, Shaw RK, Hogan JF, Holschuh KR. Long term ventilatory support by diaphragm pacing in quadriplegia. Ann Surg 1976; 183:566-77. 